\title{
Hebammenmangel in deutschen Kreißsälen - Doulas füllen die Lücke
}

\author{
Melanie Hartmann
}

Durch den Fachkräftemangel fehlen Hebammen in den Kreißsälen der Krankenhäuser, aber auch freiberufliche Hebammen sind zunehmend rar. Deshalb steht vielen Frauen nicht die Betreuung zur Verfügung, die sie sich während und nach der Geburt wünschen. Die Rolle der Doulas wird dadurch immer wichtiger. Dieser Beitrag widmet sich dem aktuellen Forschungsstand zur Bedeutung der Doulas und setzt sich mit Konflikten und Potenzialen in der Zusammenarbeit mit Hebammen auseinander.

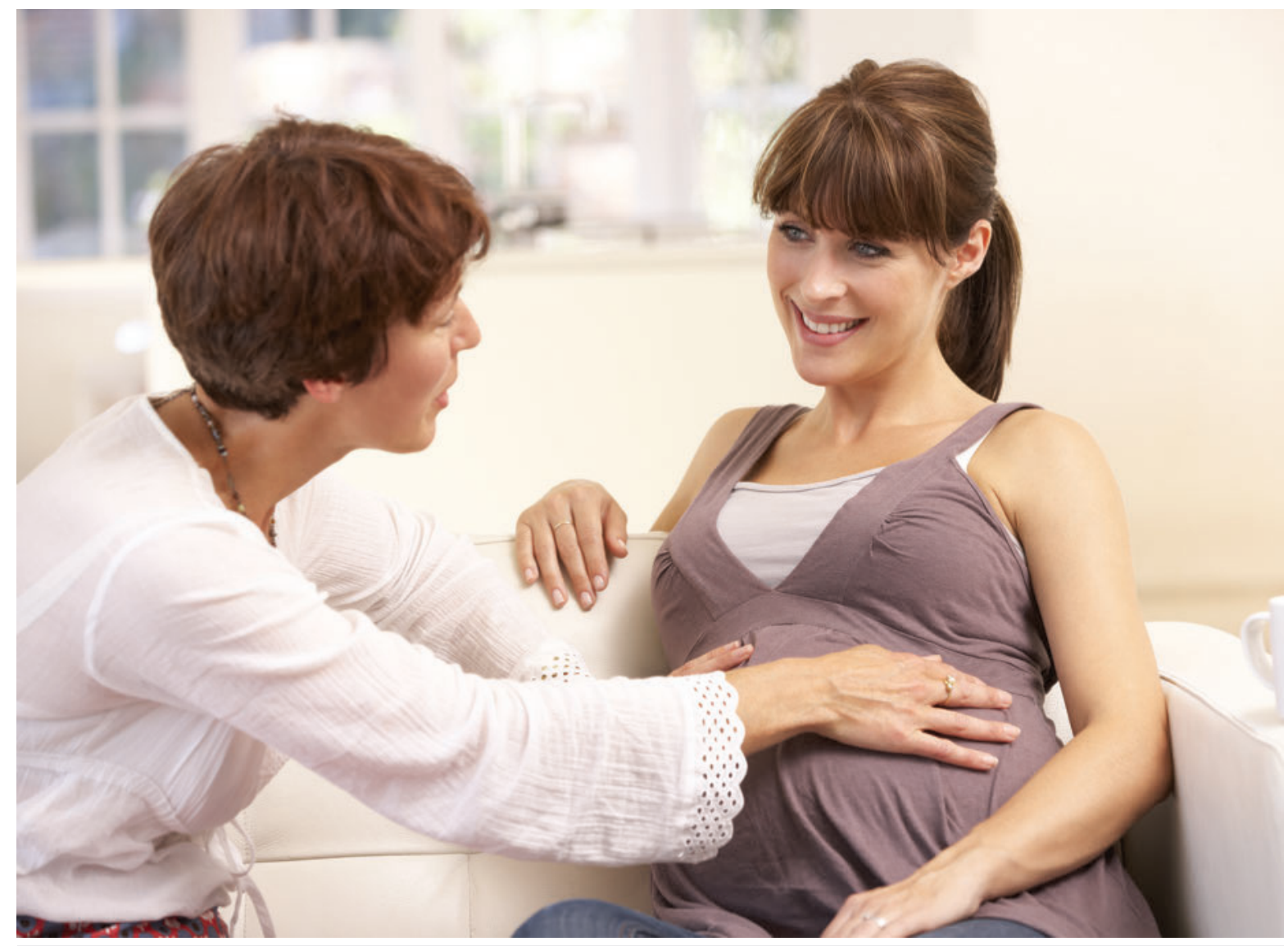

Abb. 1 Doulas bieten den Frauen, die sie betreuen, kontinuierliche Begleitung während der Schwangerschaft und unterstützen sie während und nach der Geburt. (Foto: stock.adobe.com - Monkey Business, Symbolbild)

Steigende Geburtenzahlen, Stellenabbau als Einsparungspotenzial, zunehmende administrative Aufgaben und umfassendere Dokumentationsaufgaben aufgrund forensischer Aspekte haben in Deutschland zu einem Hebammenmangel in den Krankenhäusern, aber auch in der Freiberuflichkeit geführt [10]. 
Frauen haben während ihrer Schwangerschaft, während der Geburt und post partum einen gesetzlichen Anspruch auf Hebammenhilfe ( $\$ 24 d$ SGBV). Eine Präzisierung der geforderten Betreuungszeit sub partu bleibt dabei jedoch aus [14].

Die Lücke zwischen benötigter und gewünschter Betreuung von den Frauen bzw. Paaren im Kreißsaal und der geleisteten Betreuung klafft weit auseinander und wird immer größer. In einer Studie von Stengel und Borde berichteten $55 \%$ der 567 befragten Mütter, die ihre Kinder in Berliner Kliniken geboren hatten, dass die Hebamme unter $50 \%$ der Zeit von der Aufnahme bis zum Verlassen des Kreißsaals anwesend war. $41 \%$ Prozent der befragten Frauen empfanden dabei den Betreuungsumfang der Hebamme als zu gering [14].

Doulas können diese Lücke füllen. Sie werden von der Frau engagiert und lernen sie schon während der Schwangerschaft kennen. Die Doulas begleiten die Frau zur Geburt und bieten so eine kontinuierliche Betreuung, zeigen Strategien und Techniken zur Schmerzverarbeitung, ermutigen die Gebärende und fördern die Kommunikation mit dem Fachpersonal [11].

\section{Geschichte der Doulas}

Die Geschichte der Doulas begann in den 1980er-Jahren in den USA. Aufgrund steigender Kaiserschnittraten und Interventionen während der Geburt, begannen die Frauen sich nach Unterstützerinnen umzusehen, die sie während der Geburt begleiten und vor unnötigen Interventionen schützen. Die Rolle der Doula gewann so immer mehr Bekanntheit und Ansehen [9].

Einflussfaktoren auf das Geburtsoutcome wurden vermehrt beforscht und die Studien kamen zu dem Schluss, dass eine kontinuierliche Betreuung der Frauen während der Geburt Interventionen sub partu deutlich reduzieren kann [9]. Seitdem steigt die Bedeutung der Doulas stetig, nicht nur in den USA, sondern zunehmend auch international. Dabei sind jedoch die angebotenen Leistungen so verschieden wie die Gesundheitssysteme der Länder. In Ländern, in denen eine postpartale Betreuung durch Hebammen abgesichert ist, finden sich Doulas hauptsächlich in der Betreuung sub partu wieder. Meist sind Doulas selbstständig. In den USA gibt es jedoch auch bereits in Krankenhäusern angestellte Geburtsbegleiterinnen [5].

Genaue Zahlen zu arbeitenden Doulas gibt es weder für Deutschland noch für andere Länder. Da Doula keine geschützte Berufsbezeichnung ist und es keine Registrierungspflicht gibt, kann die Zahl der arbeitenden Doulas nur anhand der Mitgliederzahlen in den Vereinen und den Zahlen der Zertifizierungen geschätzt werden.
So findet man beim Verein Doulas in Deutschland e. V., der 2008 gegründet wurde, 173 Doulas über ganz Deutschland verteilt [8]. 700 registrierte Doulas finden sich im Verein Doula UK, dem seit 2001 zertifizierte Doulas beitreten können [7]. Der 1992 gegründete U.S. amerikanische Verein DONA International zählt hingegen 13000 durch ihn zertifizierte Doulas aus 56 Ländern [6].

\section{Kontinuierliche Betreuung während der Geburt - egal durch wen?}

Die kontinuierliche Betreuung wird in der Literatur recht heterogen definiert. Bohren et al. haben den Begriff in ihrem Review zur kontinuierlichen Betreuung sub partu im Sinne der Eins-zu-Eins-Betreuung definiert. Das bedeutet, eine Betreuungsperson steht von der frühen Eröffnungsperiode bis mindestens zur Geburt allein der Frau bzw. dem Paar zur Verfügung und bietet emotionale Unterstützung, Komfortmaßnahmen sowie auch Informationen zum Geburtsfortschritt und Beratung zu Bewältigungsstrategien [3].

Da weniger als $2 \%$ der Geburten in Deutschland außerklinisch stattfinden (QUAQ), sieht sich die Mehrheit der Frauen mit einem Setting konfrontiert, in dem eine solche kontinuierliche Betreuung eher die Ausnahme als die Regel ist [3][14]. Und das obwohl positive Effekte einer Eins-zu-Eins-Betreuung mittlerweile in vielen Studien bewiesen wurden [10].

Das Cochrane Review von Bohren et al. hat die Ergebnisse von 26 Studien aus 17 Ländern (USA, Kanada, Australien, Finnland, Belgien, Frankreich etc.) mit Daten von insgesamt 15858 Frauen untersucht und Hinweise auf weniger Analgesien, niedrigere Sectioraten, seltener vaginal operative Geburten, verkürzte Geburtsdauer und eine größere Zufriedenheit mit dem Geburtserlebnis aufdecken können. Ein Einfluss der Eins-zu-Eins-Betreuung auf andere geburtshilfliche Interventionen, Komplikationen sowie auf das Stillen postpartum konnte nicht nachgewiesen werden. Das Outcome zeigte sich beeinflusst durch das geburtshilfliche Setting und Vorgehen, in dem die Eins-zu-Eins-Betreuung angeboten wurde sowie durch das Vertrauen das zwischen Frau und Betreuungsperson vorherrschte [3].

Positive Effekte in Bezug auf das Geburtsoutcome zeigten sich vor allem, wenn die Betreuungsperson keine weiteren Aufgaben hatte, nicht in der Geburtsklinik angestellt war und nicht aus dem sozialen Umfeld der Frauen kam [3]. Die anwesenden Personen aus dem sozialen Umfeld der Frauen brachten sich häufiger unterstützend in das Geburtsgeschehen ein, wenn eine Fachperson kontinuierlich anwesend war. Insgesamt hatte die Anwesenheit von vertrauten Personen aus dem sozialen Umfeld einen schützenden Effekt vor 
negativen Geburtserfahrungen [3][4]. Auch wenn diesen Ergebnissen durch die Herausgeber des Reviews nur eine geringe Evidenz zugesprochen wurde, zeigt dies die große Bedeutung der kontinuierlichen Betreuung, auch in Hinblick darauf, dass keinerlei negative Konsequenzen für eine Eins-zu-Eins- Betreuung gefunden wurden.

Damit ist deutlich geworden, dass eine kontinuierliche Betreuung durch Personen mit Fachwissen einen positiven Effekt auf den Geburtsverlauf hat. Die Frage, wer genau die kontinuierliche Betreuung anbieten soll, ist nur wenig untersucht worden und nicht endgültig geklärt.

Die kontinuierliche Betreuung durch eine Doula bringt bestimmte Vorteile mit. Dazu gehört, dass durch die Frauen engagierte Doulas keine weiteren Verpflichtungen haben und sich Schwangere und Doula häufig im Vorfeld der Geburt kennenlernen und bereits präpartal eine gute Vertrauensbasis aufbauen können. Für ausländische Frauen stellen sie zudem einen großen Gewinn dar, indem sie helfen können, Sprachbarrieren zu überwinden und kulturelle Unterschiede zu berücksichtigen [1][2]. Insgesamt wurden bei einer kontinuierlichen Betreuung durch eine Doula eine kürzere Geburtsdauer, weniger Interventionen und eine größere Zufriedenheit der Frauen mit ihrem Geburtserlebnis festgestellt. Die meisten Studien zu diesem Thema kommen jedoch aus den USA. Dort herrscht eine andere medizinische Versorgung, zudem wurden als Vergleichsgruppen immer Gruppen ohne kontinuierliche Betreuung oder ohne Begleitpersonen herangezogen. Daher kann nicht geklärt werden, ob die positiven Effekte der Doula-Betreuung oder der kontinuierlichen Betreuung im Allgemeinen zuzuschreiben sind [10].

So haben Campbell und Kollegen in ihrer randomisiert kontrollierten Studie in den USA eine kürzere Geburtsdauer auch bei Frauen gefunden, die Personen aus dem sozialen Umfeld mitbrachten, denen vorher in zwei Stunden geburtshilfliches Wissen vermittelt wurde. Die Betreuung der Kontrollgruppe wurde, wie in Nordamerika meist üblich, durch geburtshilflich ausgerichtete Krankenschwestern übernommen und die Geburt ärztlich geleitet [4]. Es wird vermutet, dass die eher medizinisch geprägte Versorgung das Outcome der Studie verstärkt hat und dass gerade in Ländern mit einer medizinisch und technisch geprägten Betreuung eine den Bedürfnissen und Wünschen der Frauen angepasste Geburt nur mit einer ergänzenden psychosozialen Betreuungsperson ermöglicht werden kann [10].

Auch in Settings, in denen das Mitbringen einer Begleitperson nicht üblich ist, konnten positive Effekte auf den Geburtsverlauf durch eine weibliche Angehörige festgestellt werden [10].
Wird eine Eins-zu-Eins-Betreuung durch Hebammen geleistet, welche die Frau im besten Fall bereits in der Schwangerschaft kennengelernt hat, oder fand sich eine geringe Arbeitsbelastung der Hebamme, konnten dieselben positiven Effekte auf das Geburtsoutcome betrachtet werden, wie in der Studie von Bohren und Kollegen [10][13].

\section{Konflikte und Potenziale der Zusammenarbeit von Doula und Hebamme}

Derzeit stellt sich in Deutschland jedoch gar nicht die Frage, welche Berufsgruppe die kontinuierliche Betreuung am besten gewährleisten soll. Das Fachpersonal wird durch den Fachkräftemangel und das Eingebundensein in klinische Strukturen und Abläufe an einer Eins-zu-EinsBetreuung gehindert. Früher oder später wird somit eine Mehrheit der in der Klinik tätigen Hebammen auf eine Frau oder ein Paar treffen, die sich für eine individuell auf sie angepasste und kontinuierliche Betreuung eine Doula mit zur Geburt bringen.

Dies birgt sowohl Konflikte als auch Potenziale für beide Berufsgruppen. In einer qualitativen Studie aus Australien von Stevens und Kollegen aus dem Jahre 2010 wurden 11 Hebammen und 6 Doulas interviewt, die jeweils schon mit der anderen Berufsgruppe zusammengearbeitet haben. Dabei wurden beide Parteien zur empfundenen Rolle einer Doula während Schwangerschaft, Geburt und Wochenbett befragt sowie Erfahrungswerte zur interdisziplinären Zusammenarbeit und Einfluss einer Doula auf die Geburtserfahrung der Frauen ermittelt [15].

\section{Sicht der Hebammen}

Die Hebammen sahen ihre Position bedroht und fühlten sich in die Rolle einer rein medizinisch arbeitenden Fachkraft gedrängt, da die kontinuierliche und emotionale Betreuung der Frauen von den Doulas gewährleistet wurde. Zudem schilderten die befragten Hebammen das Gefühl, dass sie weniger Vertrauen der Frauen entgegengebracht bekamen, wenn eine Doula anwesend war [15].

Aus Sicht der befragten Hebammen übertreten Doulas ihre Zuständigkeiten, indem sie Informationen und Ratschläge zum weiteren Vorgehen während der Geburt geben, während sie als Hebammen die volle Verantwortung für das Outcome der Geburt besitzen [15].

\section{Sicht der Doulas}

Die Doulas äußerten, häufig mit einer ablehnenden Haltung der Hebammen konfrontiert worden zu sein und 
gaben an, dass sie keinesfalls den Hebammen den Rang ablaufen wollen, sondern mit ihnen fühlen, weil sie an das schlechte Gesundheitssystem gebunden sind. Sie wollen die Hebammen unterstützen, um gemeinsam eine frauenzentrierte Betreuung zu gewährleisten [15].

Insgesamt kam die Unterstützung durch die Doulas bei den Hebammen gut an und sie unterstrichen die Zurückhaltung der Doulas, wenn diese merkten, dass sie gerade nicht benötigt werden [15].

In einer qualitativen Untersuchung aus Schweden wurden zehn Hebammen befragt, die zugewanderte Frauen während der Geburt zusammen mit einer Doula betreuten. In diesem Fall wurden Doulas als Unterstützung und Vermittlerin angesehen. Sie fungierten nicht nur als Dolmetscherinnen, sondern gaben auch Hinweise auf und Erklärungen zu kulturellen Unterschieden. Des Weiteren haben Doulas die Möglichkeit, die Befindlichkeiten und Wünsche der Frauen im Vorfeld der Geburt kennenzulernen und so den Frauen zusammen mit den Hebammen zu individuell angepassten positiven Geburtsverläufen zu verhelfen [1].

\section{Sicht der Gebärenden}

Frauen, die eine Doula mit zur Geburt brachten und eine Abneigung der Fachperson gegenüber dieser bemerkten, empfanden die Beziehung zur Fachperson als angespannt, konfrontativ und gaben teilweise an, keinen Bezug zur Fachperson aufbauen zu können. Wenn die Frauen die Zusammenarbeit zwischen Doula und Fachperson als produktiv empfanden, wurde von diesen auch ein positives und bestärkendes Geburtserlebnis geäußert. Im Gegenzug wurden von den Frauen Konflikte zwischen Fachperson und Doula mit einem negativen Geburtserlebnis in Zusammenhang gebracht [11]. Auch wenn diese qualitative Studie nur ein recht kleines Sample von neun Frauen aufweist, wird deutlich, dass ein Konflikt zwischen Doula und Hebamme oder anderweitiger Fachperson, egal aus welchen Gründen, immer auf dem Rücken der zu betreuenden Frauen ausgetragen wird.

\section{Weiterer Forschungsbedarf}

In Deutschland muss die Effektivität der Eins-zu-EinsBetreuung weiter beforscht werden, da sich internationale Studien aufgrund der unterschiedlichen Gesundheitssysteme nur bedingt auf deutsche Verhältnisse übertragen lassen. Auch die Rolle der Doula ist bisher in Deutschland wenig untersucht und beforscht worden. Literatur zur Wochenbettbetreuung durch Doulas und Auswirkungen dieser ist nur wenig vorhanden und stammt meist aus dem nordamerikanischen Raum, in dem keine umfassende Betreuung im Wochenbett angeboten wird [5]. 


\section{FAZIT}

Die Entstehung des Berufszweiges der Doula und deren steigende Bedeutung sind das Ergebnis eines mangelhaften Gesundheitssystems, welches den Frauen vor allem während der Geburt nicht gerecht wird. Die steigenden Geburtenzahlen kollidieren mit offenen Stellen in den deutschen Kreißsälen und überarbeiteten Hebammen, die anstatt eine Eins-zuEins-Betreuung zu gewährleisten häufig für drei Frauen gleichzeitig verantwortlich sind sind [14]. Die Studien belegen, wie wichtig eine kontinuierliche Betreuung während der Geburt für die Frauen, aber auch für ihre Partner ist. Dabei konnten die größten Effekte bei Frauen gefunden werden, die keine Begleitperson zur Geburt mitbrachten und in den USA lebten [10]. In die Lücke der gewünschten und tatsächlich erbrachten Betreuung passen die Aufgaben einer Doula perfekt, um eine optimale intrapartale Betreuung gewährleisten zu können. Doulas und Hebammen verfolgen das gleiche Ziel: Sie möchten die optimale Versorgung während der Geburt und die Zufriedenheit der Frauen mit ihren Geburtserlebnissen gewährleisten. Daher ist es in Zeiten des Fachkräftemangels unumgänglich, dass beide Berufsgruppen zusammenfinden, kooperieren und im Sinne der Frauen handeln. Es ist für beide Seiten wichtig, eine Beziehung zu entwickeln, die auf gegenseitigem Respekt und Unterstützung der unterschiedlichen Rollen basiert. Es sollten Treffen mit Hebammen und Doulas veranstaltet werden, die in der gleichen Region arbeiten. Denn die Studien zeigten, dass Hebammen und Doulas dieselben Ziele verfolgen, es häufig aber an Kommunikation zwischen beiden Berufsgruppen mangelte [11].

\section{Autorinnen / Autoren}

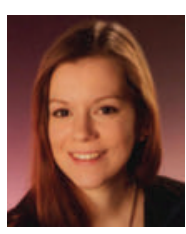

Melanie Hartmann ist Hebamme am St. Joseph Krankenhaus Berlin-Tempelhof. Über den Studiengang Hebammenkunde erwarb sie 2018 den Bachelorabschluss an der Evangelischen Hochschule Berlin.

\section{Korrespondenzadresse}

E-Mail: melie.hart@gmail.com

\section{Literatur}

[1] Akhavan Sh, Lundgren I. Midwives' experiences of doula support for immigrant women in Sweden- A qualitative study. Midwifery 2012; 28: 80-85

[2] Bohren MA, Berger BO, Munthe-Kaas $\mathrm{H}$ et al. Perceptions and experiences of labour companionship: a qualitative evidence synthesis (Review). Cochrane Database of Systematic Reviews 2019; 3 : No.: CD012449

[3] Bohren MA, Hofmeyr G], Sakala C et al. Continuous support for women during childbirth (Review). Cochrane Database of Systematic Reviews 2017; 7; Art. No.: CD003766

[4] Campbell D, Lake M, Falk M et al. A Randomized Control Trial of Continuous Support in Labor by a Lay Doula. JOGNN 2006; 35: 456-464

[5] Campbell-Voytal K, Fry McComish J, Visger JM et al. Postpartum doulas: Motivations and perceptions of practice. Midwifery 2011; 27: 214-221

[6] DONA International. About DONA International. Letzter Zugriff am 18.09.19. https: / /www.dona.org/the-dona-advan tage/about/

[7] Doula UK. About Doula UK. Letzter Zugriff am 18. 09.19. htt ps: //doula.org.uk/about-doula-uk/

[8] Doulas in Deutschland e. V. Der Verein Doulas in Deutschland e. V. Letzter Zugriff am 18.09.19. https://doulas-in-deutsch land.de/der-verein/

[9] Gilliland A. Beyond Holding Hands: The Modern Role of the Professional Doula. JOGNN 2002; 31: 762-769

[10] Knape N, Schnepp W, Krahl A et al. Die Effektivität der Einszu-eins-Betreuung während der Geburt. Eine Literaturübersicht. Z Geburtsh Neonatol 2013; 217: 161-172

[11] Papagni K, Buckner E. Doula Support and Attitudes of Intrapartum Nurses: A Qualitative Study from the Patient's Perspective. J Perinat Educ 2006; 15(1):11-18

[12] QUAQ. Geburtenzahlen in Deutschland. Letzter Zugriff am 14.10.19. https://www.quag.de/quag/geburtenzahlen.htm

[13] Sandall J, Soltani H, Gates S et al. Midwife-led continuitymodels versus othermodels of care for childbearing women (Review). Cochrane Database of Systematic Reviews 2016; 4: Art. No.: CD004667

[14] Stengel V, Borde T. Hebammenpräsenz und Qualität der Betreuung bei Klinikgeburten in Berlin: Ergebnisse einer Online Befragung von Müttern. Z Hebammenwiss. 2019; 1: 26-34

[15] Stevens J, Dahlen H, Peters K et al. Midwives' and doulas'perspectives of the role of the doula in Australia: A qualitative study. Midwifery 2011; 27: 509-516

\section{Bibliografie}

DOI https://doi.org/10.1055/a-1029-5660

Die Hebamme 2019; 32: 44-48

(c) Georg Thieme Verlag KG Stuttgart · New York ISSN 0932-8122 